\title{
QRS Axis Indeterminate
}

National Cancer Institute

\section{Source}

National Cancer Institute. QRS Axis Indeterminate. NCI Thesaurus. Code C102701.

An electrocardiographic finding in which the frontal plane QRS axis cannot be calculated. 